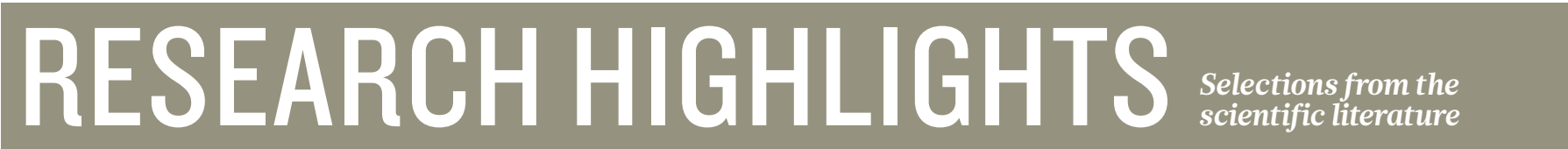

\section{INFECTIOUS DISEASE}

\section{Antibody for range of ebolaviruses}

Antibodies that recognize multiple ebolavirus species could treat the deadly infection.

Humans infected with ebolaviruses make antibodies that bind to proteins on the surface of virus particles. This prevents the virus from infecting more cells, but it is unclear whether antibodies for one of the species of ebolavirus will work for the others. To find out, Alexander Bukreyev at the University of Texas Medical Branch in Galveston, James Crowe at Vanderbilt University in Nashville, Tennessee, and their team cultured antibody-producing B cells from seven people who survived a 2007 Ebola outbreak in Uganda, caused by an ebolavirus species called Bundibugyo.

Some of the antibodies blocked two other species of ebolaviruses. One of the Bundibugyo antibodies protected guinea pigs that had been infected with another species of ebolavirus. Cell http://doi.org/bb3g (2016)

\section{ASTRONOMY}

\section{Turbulence roils Iuminous galaxy}

The brightest-known galaxy is blasting gas out into space - and providing astronomers with a rare glimpse of how extreme galaxies evolve.

Known as W2246-0526, the galaxy is as bright as 350 trillion Suns and is powered by a supermassive black hole at its heart. A team led by Tanio Díaz-Santos at Diego Portales University in Santiago, Chile, used

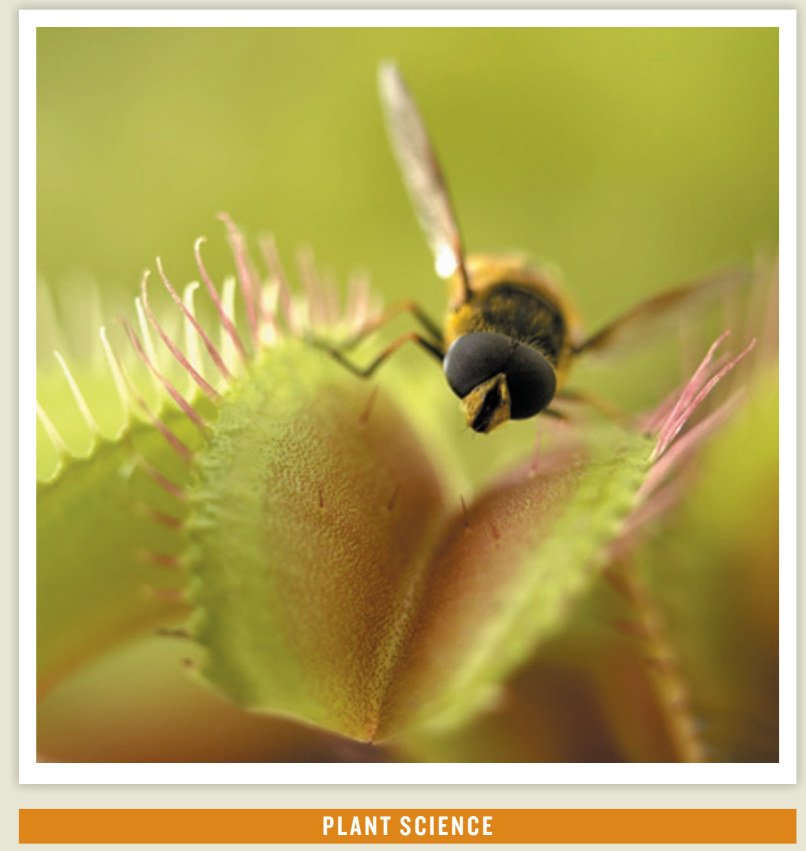

\section{Plants count to five}

Venus flytraps count the number of touches from trapped insect prey before producing digestive juices.

Erwin Neher at the Max Planck Institute for Biophysical Chemistry in Göttingen and Rainer Hedrich of the University of Würzburg, both in Germany, and their colleagues touched the leaves of Venus flytraps (Dionaea muscipula; pictured) to mimic captured, moving prey. They recorded the plant's electrical impulses in response to 1-60 touches, and found that two impulses triggered the trap to close. But only after the fifth impulse did plants begin to synthesize the digestive enzyme hydrolase and increase their production of a sodium transporter, which is used to absorb nutrients.

Venus flytraps may record the touches of potential prey to identify insects that are worth digesting, the authors say. Curr. Biol. http://doi.org/bbzp (2016)

the high-resolution

Atacama Large Millimeter/ submillimeter Array in Chile to study carbon ions rushing outwards from the galaxy.

The gas races out at speeds of about 2 million kilometres an hour, violently illuminating the surrounding space. W2246-0526 might be spewing out much of its energy, and could become more tame in the future. Astrophys. J. Lett. 816, L6 (2016)

\section{BIOENGINEERING}

\section{Shielded cells} treat diabetes

Insulin-producing cells derived from human stem cells restore blood sugar to normal levels when encased in a porous biomaterial and implanted in diabetic mice.

People with severe type 1 diabetes can sometimes be treated with a transplant of insulin-producing cells from cadavers, but the cell supply is limited. The recipient must also stay on drugs to stop the immune system attacking the cells. Instead, Daniel Anderson of the Massachusetts Institute of Technology in Cambridge and his colleagues derived insulin-producing $\beta$-cells from human embryonic stem cells and encapsulated them in a substance called TMTD alginate.

When implanted into diabetic mice, the coated cells were shielded from immune attack. The animals also maintained normal bloodsugar control until the implants were removed 174 days later.

Nature Med. http://dx.doi. org/10.1038/nm.4030 (2016)

\section{OCEANOGRAPHY}

\section{Rising seas differ by region}

The expansion of oceans as the climate warms has contributed to a rise in global sea levels of about 1.38 millimetres per year - roughly twice that of previous estimates.

Roelof Rietbroek at the University of Bonn in Germany and his colleagues analysed sea-surface heights from satellite radar data, and looked at changes in water storage from the Gravity Recovery And Climate Experiment (GRACE). They found that between 2002 and 2014, sea levels have increased in total by roughly $2.74 \mathrm{~mm}$ per year, with $1.38 \mathrm{~mm}$ of that coming from ocean thermal expansion and $1.08 \mathrm{~mm}$ from melting ice sheets, glaciers and other water sources on land, such as rivers.

The team also uncovered large regional differences. For example, the Philippines experienced a sea-level rise of about $14.7 \mathrm{~mm}$ per year, mostly because of thermal 